\title{
The impact of GJA8 SNPs on susceptibility to age-related cataract
}

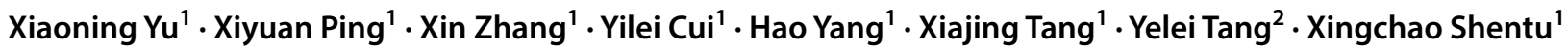

Received: 13 July 2018 / Accepted: 12 October 2018 / Published online: 22 October 2018

(C) The Author(s) 2018

\begin{abstract}
The gap junction protein alpha 8 (GJA8) gene has been widely studied in human congenital cataracts. However, little is known about its relationship with age-related cataract (ARC). In this study, three GJA8-tagged single nucleotide polymorphisms related to an increased ARC risk were identified: rs2132397 for general ARC under both dominant and additive models; rs7541950 for general ARC under both recessive and additive models; and rs6657114 for cortical cataract under the recessive model. To uncover the underlying mechanisms, this study also sought to explore whether GJA8 is involved in the autophagy process in human lens epithelial cells. The results showed that GJA8 may participate in autophagy to maintain the intracellular environment, which may be a novel mechanism for cataract formation induced by GJA8. In conclusion, this study identified the genetic susceptibility of GJA8 polymorphisms on ARC and provides new clues for fully understanding the pathological mechanism of GJA8 variants in affecting lens opacity.
\end{abstract}

\section{Introduction}

Cataracts are the leading cause of blindness and a major cause of vision impairment worldwide (Bourne et al. 2013; Congdon et al. 2003), with age-related cataract (ARC) being the most common type (Klein et al. 1992, Su et al. 2013). The precise etiology of ARC is not yet fully understood, but it is widely accepted that genetic factors play a vital role in the formation of ARC (Su et al. 2013). Studies of twins have implicated that the broad sense heritability is $58 \%$ for the cortical subtype of ACR and $48 \%$ for the nuclear subtype of ARC (Hammond et al. 2000, 2001). Genetic variations may be directly involved in the development of ARC or may increase lens susceptibility to environmental risk factors (Hammond et al. 2001). To date, over 40 different genes

Xiaoning Yu and Xiyuan Ping have contributed equally to this manuscript.

Electronic supplementary material The online version of this article (https://doi.org/10.1007/s00439-018-1945-5) contains supplementary material, which is available to authorized users.

Xingchao Shentu

stxc@zju.edu.cn

1 Eye Center, The Second Affiliated Hospital, Zhejiang University School of Medicine, Hangzhou 310009, Zhejiang, China

2 The Second Affiliated Hospital, Zhejiang University School of Medicine, Hangzhou 310009, Zhejiang, China and loci have been identified as related to congenital cataract formation (Mackay et al. 2014; Shiels and Hejtmancik 2013). The connexin genes, which account for a quarter of congenital cataract-related genes, are reported to be among the most widespread (Mackay et al. 2014).

The Gap junction protein alpha 8 (GJA8) gene, which belongs to the connexin gene family, is located at lq21.1 (CX50, MIM 600897, NG_016242.1) and consists of two exons (Dang et al. 2016; Li et al. 2013; Zhu et al. 2014). This gene encodes connexin 50, which is extensively and abundantly distributed in lens fibers and epithelial cells, as well as constituting a highly developed gap junction network vital for maintaining lens transparency (Beyer et al. 2013; Goodenough 1992; Jiang 2010). To date, more than 20 GJA8 variations have been identified in congenital cataract pedigrees worldwide, and most are inherited through autosomal dominance (Dang et al. 2016; Zhu et al. 2014). However, few studies have reported its relationship with ARC. Additionally, only one $G J A 8$ variant, c. $823 \mathrm{G}>\mathrm{A}$, which was first identified in a congenital cataract pedigree, has been reported to specifically cause this disease (Liu et al. 2011; Patel et al. 2017; Zhou et al. 2011). Thus, although GJA8 has been extensively investigated in congenital cataracts, further studies should be conducted to explore its relationship with $\mathrm{ARC}$, and the results may be diverse among different ethnicities. Although functional disturbances of gap junctions and hemichannels caused by GJA8 variants are considered to be the main pathogenesis for cataract formation (Beyer et al. 
2013; Goodenough 1992), Lichtenstein et al. reported the accumulation of mutated GJA8 proteins resulting from insufficient autophagy in HeLa cells (Lichtenstein et al. 2011), and this study speculates that this may be another pathogenic mechanism of GJA8 mutations.

Considering that tag single nucleotide polymorphisms (SNPs) make it possible to identify genetic variation and association to phenotypes without genotyping every SNP in a gene, this study aimed to fully screen GJA8-tagged SNPs in ARC patients. As the alteration of the autophagy-related proteins LC3 and P62 in GJA8-overexpressed human lens epithelial (HLE) cells was detected, this study also explores whether GJA8 participates in the autophagy process in the lens.

\section{Materials and methods}

\section{Study participants}

A total of 1190 unrelated participants, comprising 690 ARC patients and 550 control subjects, were included in this study. All participants were Han Chinese and were recruited from the Eye Center of the Second Affiliated Hospital, Medical College of Zhejiang University, Hangzhou, China. This study adhered to the tenets of the Declaration of Helsinki and was approved by the ethics committee of the Second Affiliated Hospital, Medical College of Zhejiang University, Hangzhou, China. In addition, informed consent was obtained from every participant.

All ARC patients underwent complete ophthalmic examinations, including fundus photography, the best-corrected visual acuity (BCVA) measurement, and lens examination with a slit lamp biomicroscope after mydriasis. Clinical diagnosis and classification were based on the lens opacities classification system II (Locs II) (Chylack et al. 1989). According to the degenerative regions of the lens, ARC was classified into four subtypes: cortical cataract $(C)$, nuclear cataract $(N)$, posterior subcapsular cataract (PSC), and multiple cataract $(M$, more than one cataract subtype in an eye) (Klein et al. 1992). Patients with cataracts caused by trauma, uveitis, diabetes, high myopia, and other causes were excluded.

Control subjects were recruited from healthy individuals who received routine health examinations at the Second Affiliated Hospital, Medical College of Zhejiang University, Hangzhou, China. All control subjects also underwent complete ophthalmic evaluations, which indicated transparent lens.

\section{SNP selection and genotyping}

Haplotype-tagging SNPs in the GJA8 gene were selected from the HapMap Beijing Han Chinese (CHB) population (HapMap Genome Browser release \#27, accessed April 29, 2014; available at http://hapmap.ncbi.nlm.nih.gov/). Based on the tagger-pairwise method, with an $R$ square $\left(r^{2}\right)>0.8$ and a minor allele frequency (MAF) $>0.10$, a total of 8 SNPs in GJA8 were selected. Genomic DNA was extracted from peripheral blood leukocytes from all subjects using a Simgen Blood DNA mini kit (Simgen, Hangzhou, China).

\section{Statistical analysis}

The Hardy-Weinberg equilibrium (HWE) of each SNP was assessed by the $\chi^{2}$ test using PLINK (v1.07) (available at http://pngu.mgh.harvard.edu/ purcell/plink/). All continuous variables corresponding to the subjects' characteristics were summarized as mean \pm SD. The association between the SNPs and ARC was examined under three different genetic models: dominant, recessive, and additive. Allelic distributions were compared between the ARC patients and the control subjects using the $\chi^{2}$ test. A logistic regression analysis was conducted to evaluate the genetic effects of the GJA8 SNPs after adjusting for age and sex. The Bonferroni correction for multiple testing was used to reduce the rate of type I error (We have tested 57 different SNPs in the same case and control groups in all. And GJA8 tag SNPs are only part of them, thus the corrected significant level was set to be $0.05 / 57 * 3$ ). An Armitage trend test was performed for the risk SNPs identified by the logistic regression analysis in the additive model using SAS software. All the other statistical analyses were conducted using SPSS software, version 11.0. A two-tailed $p$ value $<0.05$ was considered statistically significant, otherwise indicated.

\section{Construction of plasmids}

The human GJA8 coding sequence was acquired by the polymerase chain reaction (PCR) with the primers: sense primer 5'-CTCGAGATATGGGCGACTGG-3', and antisense primer 5'- CAGAATTCTCATACGGTTAG-3'. The PCR products and vector pEGFP-C1 were digested by XhoI and EcorI (Takara, Japan). The construction was confirmed through direct sequencing. Transient transfection of pEGFP-C1-GJA8 was carried out using the Lipofectamine 2000 reagent (Invitrogen, Carlsbad, CA, USA) according to the manufacturer's protocols.

\section{Cell culture}

The HLE cells (SRA 01-04) (Ibaraki et al. 1998) were obtained from the RIKEN Cell Bank (Tsukuba, Japan) and 
cultured in DMEM/F12 with 10\% heat-inactivated fetal calf serum (Biological Industries, Israel) at $37{ }^{\circ} \mathrm{C}$ in a humidified atmosphere containing $5 \% \mathrm{CO}_{2}$. All cell culture medium components were purchased from Invitrogen Life Technologies. Cells were prepared, and washed with PBS twice, then replaced with no fetal calf serum medium for $2 \mathrm{~h}$ or $4 \mathrm{~h}$ as was done for the Sta2h group or Sta4h group, or replaced with complete medium for $2 \mathrm{~h}$ as was done for the $\mathrm{NC}$ group.

\section{Immunofluorescence}

HLE cells were adhered overnight in 24-well plates to grow to $80 \%$ cell density, then transfection with or without $1.0 \mu \mathrm{g} /$ well peGFP-C1-GJA8 plasmid for $24 \mathrm{~h}$, and washed with PBS for twice, replaced with no fetal calf serum media for $2 \mathrm{~h}$ or $4 \mathrm{~h}$ (Sta2h/Sta4h group) or complete medium (NC group) for $2 \mathrm{~h}$. Then the cells were washed with PBS for twice, and fixed with $4 \%$ paraformaldehyde in PBS for 10 min, washed with PBS for three times. Then, cells were blocked with $2 \%$ BSA in PBST $(0.1 \%$ Triton X-100 in PBS) for $1 \mathrm{~h}$ and incubated with primary antibody for $2 \mathrm{~h}$ [GJA8(sc-50432) 1:200 from Santa Cruz, LC3(2775) 1:200 from Cell Signaling Technology], and washed with PBST for triple; second antibody for $1 \mathrm{~h}$ (Alexa Fluor488-conjugated antibodies, Alexa Fluor555-conjugated antibodies, and DAPI from Life Technologies), and washed with PBST for triple. Images were captured using an Olympus FluoView 1000 confocal microscope at $40 \times$.

The GFP plasmid vector was used to test the possible alterations of the autophagic proteins in the IF, and no differences between in the GFP plasmid vector and the normal control were observed (Supplementary Figure S1). Thus, in our paper, we use the normal group (the NC group) as the control to explain the alteration of autophagic proteins with the overexpression of GJA8, and to prove that the autophagy process occurred in human lens epithelial (HLE) cells.

\section{Western blot}

HLE cells were adhered overnight in 6-well plates to grow to $80 \%$ cell density, then transfection with or without 1.0 or $2.0 \mu \mathrm{g} /$ well peGFP-C1-GJA8 plasmid for $24 \mathrm{~h}$, and washed with PBS for twice, replaced with no fetal calf serum media for $2 \mathrm{~h}$ or $4 \mathrm{~h}$ (Sta2h/Sta4h group) or complete medium (NC group) for $2 \mathrm{~h}$. Then the cells were lysed in lysis buffer (Shenggong, Shanghai, China) and blocked in 5\% BSA (Shenggong, Shanghai, China) in TBST $(0.1 \%$ Tween-20 in TBS). Immunocomplexes were separated by $10 \%$ SDSPAGE (sodium dodecyl sulfate-polyacrylamide gel electrophoresis) and then subjected to immunoprecipitation with the primary antibody [P62 (ab56416) 1:1000, $\alpha$-Tubulin (ab4074) 1:1000 from Abcam], and the HPR-conjugated second antibody.

\section{Results}

\section{Demographic characteristics of the participants}

A total of 690 ARC patients $(C=131, N=126, \mathrm{PSC}=73$, $M=360$ cataract patients) and 500 healthy control subjects were included in this study. No significant differences were detected between the two groups $(p>0.05)$. The general demographic characteristics of the 1190 participants are summarized in Table 1.

\section{The bioinformatics characteristics of tag SNPs}

Eight tag SNPs in GJA8 were selected for genotyping; their bioinformatics characteristics are summarized in Table 2 . No SNPs deviated from the HWE in this study.

\section{The association between tag SNPs and risk of ARC}

Two tag SNPs (rs2132397, $p_{\mathrm{a}}=9.25 \times 10^{-3}$, OR 1.413, CI $1.195-1.671, p$ for the Armitage trend test: $3 \times 10^{-4}$; rs7541950, $p_{\mathrm{a}}=0.03$, OR 1.543 , CI $1.230-1.935, p$ for the Armitage trend test: $<1 \times 10^{-4}$ ) showed a significant association with ARC risk under the additive model (Table 3). One tag SNPs (rs2132397, $p_{\mathrm{a}}=0.029$, OR 1.638, CI 1.267-2.118) showed a significant association with ARC risk under the dominant model, and one tag SNPs $\left(\mathrm{rs} 7541950, p_{\mathrm{a}}=2.33 \times 10^{-4}\right.$, OR 2.076, CI 1.543-1.047) showed a significant association with ARC risk under the recessive model. The association remained significant after correcting for multiple testing and adjusting for age and gender. A stratification analysis was also performed to explore the relationship between these SNPs and the different subtypes of ARC. Only one tag SNPs (rs6657114, $p=2.94 \times$ $10^{-5}$, OR 3.295 , CI $1.883-5.765$ ) were identified as a significant risk factor for cortical cataract under the recessive model.

Table 1 The general demographic characteristics of the subjects involved in this study

\begin{tabular}{|c|c|c|c|c|c|}
\hline \multirow[t]{2}{*}{ Group } & \multirow[t]{2}{*}{ Number } & \multicolumn{2}{|l|}{ Gender } & \multicolumn{2}{|l|}{ Age } \\
\hline & & Male (\%) & Female (\%) & Mean \pm SD & Range \\
\hline Control & 500 & 58.8 & 41.2 & $64.306 \pm 7.586$ & 49-92 \\
\hline$A R C$ & 690 & 47.83 & 52.17 & $66.323 \pm 9.781$ & $43-91$ \\
\hline C & 131 & 34.35 & 65.65 & $67.75 \pm 8.383$ & $43-88$ \\
\hline$N$ & 126 & 38.10 & 61.90 & $68.259 \pm 9.856$ & $45-87$ \\
\hline$P S C$ & 73 & 45.21 & 54.79 & $66.484 \pm 10.274$ & $45-90$ \\
\hline$M$ & 360 & 56.11 & 43.89 & $65.170 \pm 9.936$ & $38-91$ \\
\hline
\end{tabular}

$A R C$ age-related cataract, $C$ cortical cataract, $N$ nuclear cataract, $P S C$ posterior subcapsular cataract, $M$ multiple cataract 
Table 2 The bioinformatics characteristics of the involved eight GJA8 SNPs
Table 3 The relationship between GJA8 tag SNPs and ARC risk

\begin{tabular}{lllllll}
\hline SNPs & Minor allele & Call rate & MAF & $\begin{array}{l}\text { Test for HWE } \\
(p \text { value })\end{array}$ & Control (MAF) & ARC (MAF) \\
\hline rs6657114 & T & 0.99916 & 0.360 & 0.6317 & 0.326 & 0.385 \\
rs6688578 & C & 0.99916 & 0.108 & 0.4799 & 0.096 & 0.118 \\
rs7544630 & C & 0.99916 & 0.235 & 0.4026 & 0.243 & 0.229 \\
rs1532399 & A & 0.99916 & 0.319 & 0.6951 & 0.288 & 0.342 \\
rs2132397 & G & 0.99916 & 0.176 & 0.3105 & 0.14 & 0.202 \\
rs7541950 & T & 0.99916 & 0.474 & 0.1405 & 0.43 & 0.493 \\
rs9437981 & G & 0.99916 & 0.359 & 0.7941 & 0.327 & 0.382 \\
rs6674829 & G & 0.99916 & 0.415 & 0.1275 & 0.385 & 0.436 \\
\hline
\end{tabular}

\begin{tabular}{|c|c|c|c|c|}
\hline \multirow[t]{2}{*}{ SNP } & \multirow[t]{2}{*}{ Genetic model } & \multirow[t]{2}{*}{$\chi^{2}$ test $\left(p / p_{\mathrm{a}}\right)$} & \multicolumn{2}{|l|}{ Logistic regression } \\
\hline & & & $p / p_{\mathrm{a}}$ & OR $(95 \% \mathrm{CI})$ \\
\hline \multirow[t]{3}{*}{ rs6657114 } & Dominant & 0.067 & - & - \\
\hline & Recessive & 0.002 & - & - \\
\hline & Additive & 0.005 & - & - \\
\hline \multirow[t]{3}{*}{ rs6688578 } & Dominant & 0.062 & - & - \\
\hline & Recessive & 0.978 & - & - \\
\hline & Additive & 0.166 & - & - \\
\hline \multirow[t]{3}{*}{ rs7544630 } & Dominant & 0.428 & - & - \\
\hline & Recessive & 0.522 & - & - \\
\hline & Additive & 0.669 & - & - \\
\hline \multirow[t]{3}{*}{ rs 1532399} & Dominant & 0.053 & - & - \\
\hline & Recessive & 0.003 & - & - \\
\hline & Additive & 0.008 & - & - \\
\hline \multirow[t]{3}{*}{ rs2132397 } & Dominant & $4.50 \times 10^{-5} / 7.70 \times 10^{-3}$ & $1.68 \times 10^{-4} / 0.029$ & $1.638(1.267-2.118)$ \\
\hline & Recessive & 0.156 & - & - \\
\hline & Additive & $2.25 \times 10^{-4} / 0.038$ & $5.41 \times 10^{-5 /} / 9.25 \times 10^{-3}$ & $1.413(1.195-1.671)$ \\
\hline \multirow[t]{3}{*}{ rs7541950 } & Dominant & 0.093 & - & - \\
\hline & Recessive & $1.20 \times 10^{-5} / 2.05 \times 10^{-3}$ & $1.36 \times 10^{-6} / 2.33 \times 10^{-4}$ & $2.076(1.543-1.047)$ \\
\hline & Additive & $7.00 \times 10^{-5} / 0.012$ & $1.78 \times 10^{-4} / 0.030$ & $1.543(1.230-1.935)$ \\
\hline \multirow[t]{3}{*}{ rs9437981 } & Dominant & 0.074 & - & - \\
\hline & Recessive & 0.004 & - & - \\
\hline & Additive & 0.011 & - & - \\
\hline \multirow[t]{3}{*}{ rs6674829 } & Dominant & 0.216 & - & - \\
\hline & Recessive & 0.003 & - & - \\
\hline & Additive & 0.011 & - & - \\
\hline
\end{tabular}

\section{The alteration of autophagy in HLE cells caused by GJA8}

To prove whether starvation can induce autophagy to detectable levels in HLE cells, the alteration of the autophagic protein LC3, which is necessary in the elongation and formation process of autophagosome, therefore used to represent the level of autophagy (Jiang and Mizushima 2015), was tested under both normal and starved conditions via immunofluorescence and western blot (Fig. 1a, b), and the results revealed that the LC3-II were significantly increased under starved conditions (Fig. 1c). LC3 puncta were detected after transfecting peGFP-C1GJA8 plasmid into HLE cells via immunofluorescence. And it was found that the LC3 puncta decreased in GJA8overexpressed HLE cells, even under starved conditions (Fig. 1d, e). To further confirm these results, this study also detected the expression of P62, which recruited by LC3 and involved in the elongation process, and negatively related with the level of autophagy (Jiang and Mizushima 2015; Pankiv et al. 2007), via western blot (Fig. 1f). In NC group, a significantly higher expression of P62 was 
A

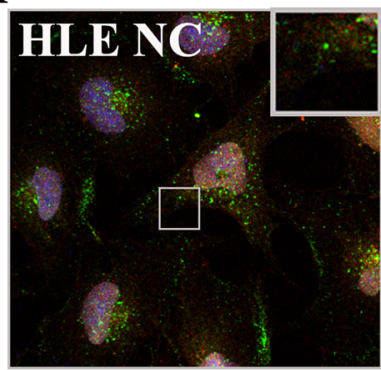

HLE Sta2h

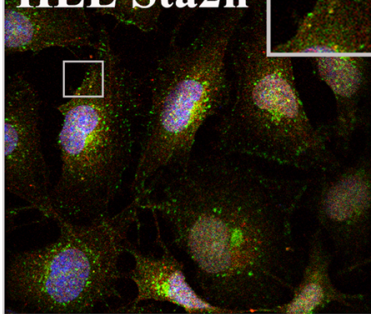

D

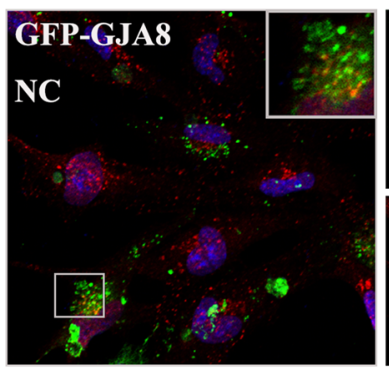

$\mathbf{E}$
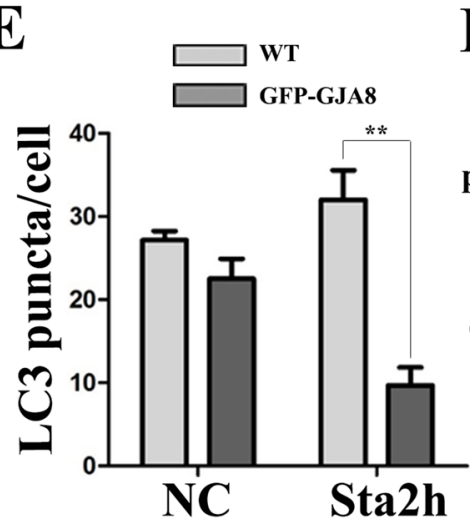
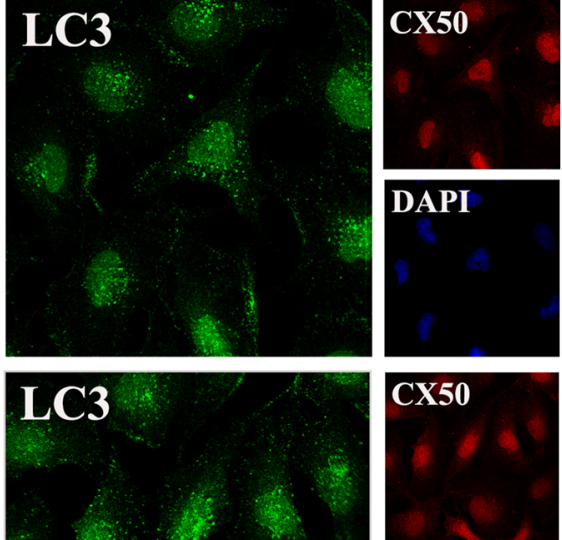

DAPI

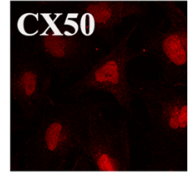

DAPI

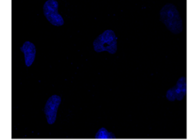

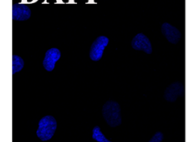
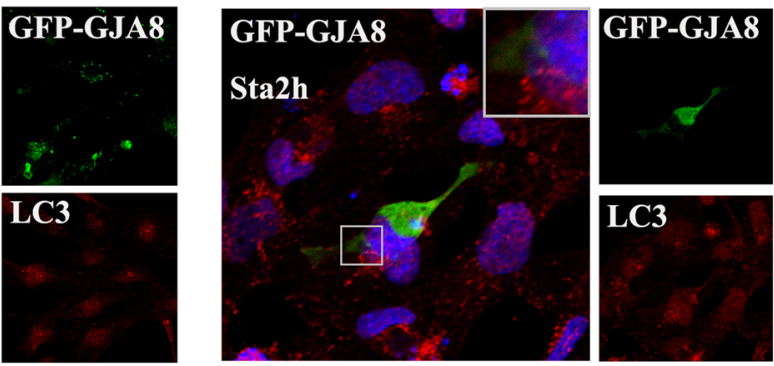

C

B
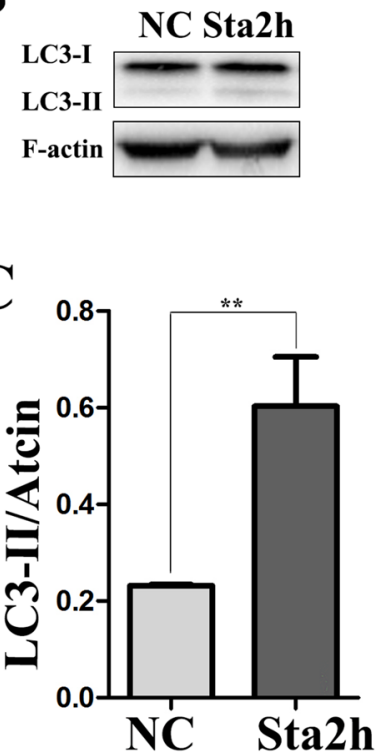

$\mathbf{F}$

Sta4h Sta2h NC NC NC

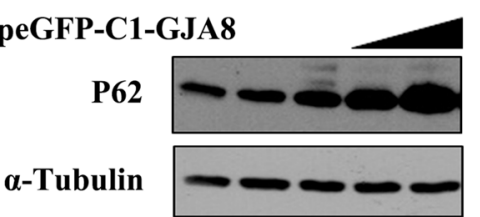

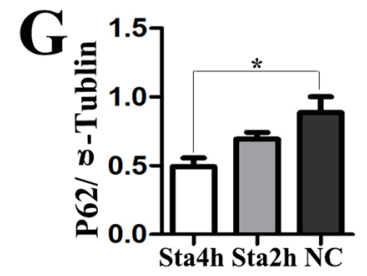

$\mathbf{H}$

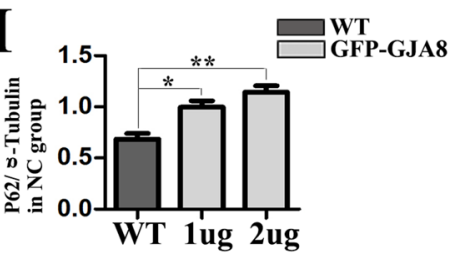

Fig. 1 Overexpressed GJA8 protein modulated the level of LC3 and P62 in HLE cells. a HLE cells stained for GJA8 (red) and LC3 (green) in the NC and Sta2h groups. b Western blot with LC3-I/II in $\mathrm{NC}$ and Sta2h groups in HLE cells. $\mathbf{c}$ The average level of LC3-II/ Actin of three independent experiments. d Transfected with peGFPC1-GJA8 in the NC and Sta2h groups, HLE cells stained for LC3 (red). e Mean number of LC3 puncta for each treatment ( $n=3$ wells, 3 independent experiments, $>50$ cells per experiment). f HLE cells for corresponding treatment and transfected with peGFP-C1-GJA8 for 0ug or $1 \mathrm{ug}$ or $2 \mathrm{ug}$, testing the expression of P62 and $\alpha$-Tubulin. $\mathrm{g}$ The average level of P62/ $\alpha$-Tubulin of three independent experiments in $\mathrm{Sta} 4 \mathrm{~h} / \mathrm{Sta} 2 \mathrm{~h} / \mathrm{NC}$ group. $\mathbf{h}$ The average level of P62/ $\alpha$-Tubulin of three independent experiments transfected with peGFP-C1-GJA8 for 0ug or $1 \mathrm{ug}$ or $2 \mathrm{ug}$ in NC group. All values are represented as the mean $+\mathrm{SEM} ; * p<0.05, * * p<0.01$ indicate significant differences with corresponding groups. Nuclei are stained with DAPI 
detected after transfected with 1.0 and 2.0 ug peGFP-C1GJA8 plasmid (Fig. 1h).

\section{Discussion}

The GJA8 protein is the major component of gap junction channels and hemichannels in the lens (Beyer et al. 2013; Goodenough 1992). To date, no study has thoroughly explored the relationship between the GJA8-tagged SNPs and ARC. Thus, this study intends to uncover the association between GJA8-tagged SNPs and ARC in the Chinese population. In the present study, a potential relationship between three GJA8-tagged SNPs (rs2132397, rs7541950 and rs6657114) and ARC is identified. Moreover, using immunofluorescence and western blot, it is found that GJA8 is involved in autophagy in HLE cells, which indicates another mechanism by which genetic variations in GJA8 could lead to cataract formation.

As mentioned above, the GJA8 gene has always been considered a cataract-related gene. More than 20 GJA8 mutations have been detected in congenital cataract pedigrees of different populations thus far (Beyer et al. 2013; Dang et al. 2016; Goodenough 1992; Graw et al. 2009; Jiang 2010; Li et al. 2013; Liu et al. 2011; Patel et al. 2017; Ren et al. 2017; Shiels and Hejtmancik 2013; Zhao et al. 2017; Zhou et al. 2011; Zhu et al. 2014). However, few studies have reported the relationship of GJA8 mutations with ARC. In general, variants in lens proteins, which would cause protein to aggregate rapidly and directly, tend to result in congenital cataracts. In contrast, variants that merely increase susceptibility to environmental risk factors usually contribute to age-related cataracts (Shiels 2017). It is worth noting that
GJA8 variants are risk factors for both types of cataracts. Moreover, GJA8 variants related to congenital cataracts could occur in any domain of the GJA8 protein (Fig. 2, Supplementary Table S1).

In the present study, for total ARC, rs2132397 and rs7541950 are found to confer 1.41- and 1.54-fold increased risks under the additive model, respectively. Also, rs2132397 is found to increase ARC risk under the dominant model, and rs7541950 is found to confer a 2.08-fold increased ARC risk under the recessive model. In addition, for ARC subtypes, only rs6657114 is found to be a potential risk factor for cortical cataract under the recessive model. Yet, for congenital individuals with GJA8 variants, nuclear cataract is the most common subtype. The results of this study indicate that GJA8 variants may induce diverse cataract subtypes. Additionally, the rs 2132397 is located in the 3 '-terminal untranslated region (3'-UTR) region of GJA8, which indicates its potential role in the interference of translation initiation. The functional consequences of the other two SNPs (rs754195 and rs6657114) are still unknown, as these two polymorphisms are located in a non-coding intron. This study speculates that they may be in linkage disequilibrium with the actual disease-causing variants in $G J A 8$, or even in a nearby causative gene. The data expand the spectrum of GJA8 variants and cataract phenotypes; however, the genotype-phenotype correlation remains unclear.

For the avascular lens, lens cell survival is dependent on intercellular communication through an extensive network of gap junctions (Ren et al. 2017). Generally, gap junction channels in HLE cells consist of two connexons; one connexon comprises one hexamer of connexins, also called a hemichannel, which is mainly formed by three different connexin forms (GJA3, GJA8, and GJA1) (Beyer et al. 2013;

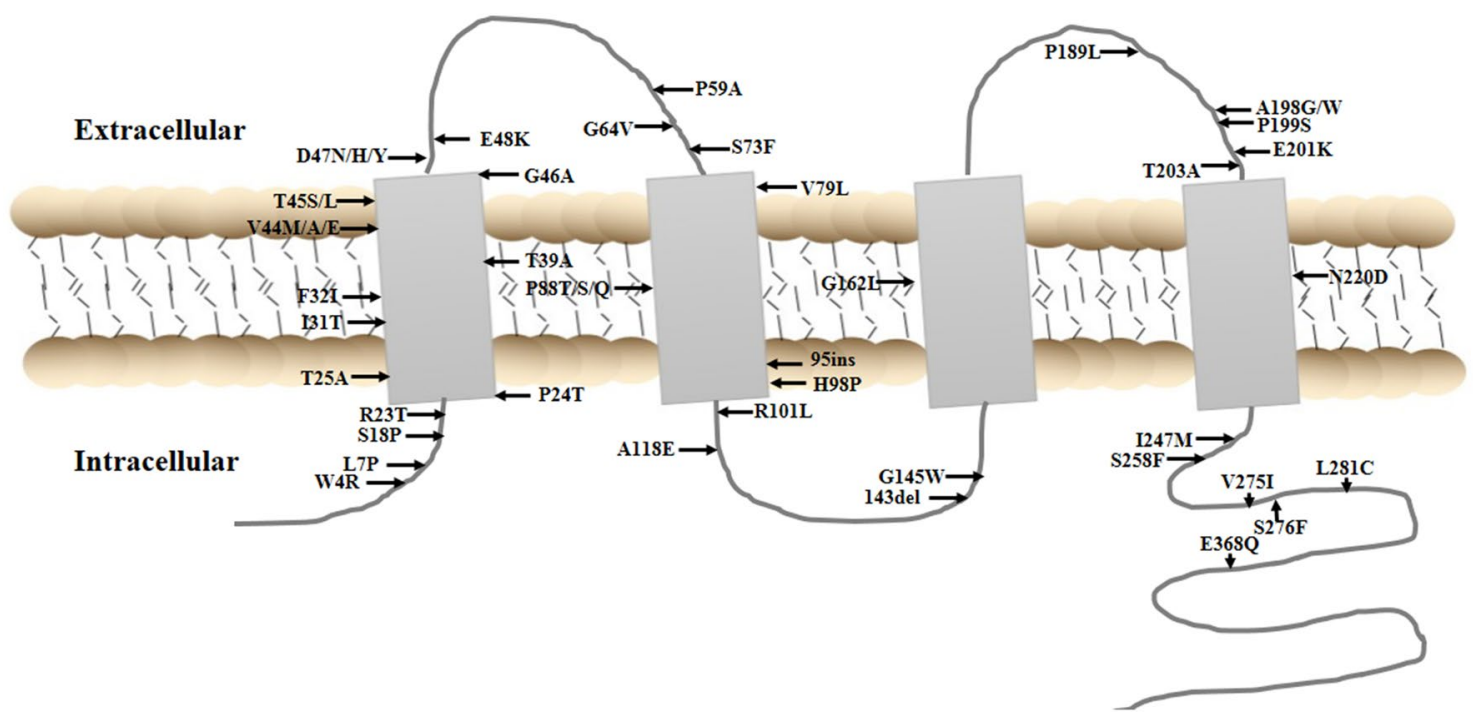

Fig. 2 Schematic diagram of GJA8 reported variants in Pubmed database 
Goodenough 1992; Zhu et al. 2014). The team for this study has reported impaired gap junctions in HLE cells with GJA8 mutations (p.V44A and p.D47H, identified from two congenital cataract families) (Li et al. 2013; Zhu et al. 2014), which is in accordance with other studies and is considered to be the main pathogenesis of congenital cataract patients with GJA8 mutations (Shiels and Hejtmancik 2013). However, several studies found that, in some congenital families with GJA8 mutations, the gap junction channel and hemichannel functions were only mildly impaired, which was insufficient to cause congenital cataracts (Graw et al. 2009). In addition, mutations in GJA8 could result in different lens morphologies and diverse patterns of lens opacities under different genetic models (Dang et al. 2016; Li et al. 2013; Patel et al. 2017; Ren et al. 2017; Zhao et al. 2017; Zhou et al. 2011). Therefore, other underlying molecular mechanisms may exist.

Autophagy is an evolutionarily conserved process that is essential for cell survival and development (Sagona et al. 2014). In lens development, autophagy participates in the degradation of cellular organelles and proteins and is crucial for transparent lens formation (Morishita and Mizushima 2016). It is worth noting that more than $80 \%$ of autophagosomes contain connexin proteins (Bejarano et al. 2014). Bejarano et al. found that connexins can form a CxAtg complex (an important complex involved in the initial step of intracellular autophagy) to modulate the formation of the autophagosomes through disturbing the formation of the complex ATG5/ATG16L/ATG12 (Bejarano et al. 2014). Lichtenstein et al. reported that as the GJA8 mutation was not enclosed by specific structures containing the autophagyrelated protein LC3, resulting in accumulation in the cytoplasm (Lichtenstein et al. 2011). Thus, this study speculates that autophagy is important for the normal turnover of GJA8 protein, which may reveal another novel mechanism for cataract formation caused by GJA8 mutation accumulation. To prove this, immunofluorescence and western blot are used to detect the alteration of the autophagic proteins LC3 and P62 in different situations as well as in normal HLE cells or overexpressed GJA8 HLE cells. LC3 is reported to be necessary in the elongation and formation process of autophagosome, which is therefore usually used to represent the level of autophagy (Jiang and Mizushima 2015). P62, recruited by LC3 and also involved in the elongation process, could be degraded by autophagosome (Pankiv et al. 2007). Thus, the level of P62 is negatively related with the level of autophagy (Jiang and Mizushima 2015; Pankiv et al. 2007). The present study finds that, in GJA8-overexpressed HLE cells, LC3 puncta decreased even under starvation conditions. which revealed overexpressed GJA8 negatively affect the process of autophagy. Consistent with this, the expression of P62 increased in GJA8-overexpressed HLE cells in NC group, compared with no transfection NC group, and this tendency was increased as the transfected with peGFP-C1-GJA8 plasmid increased. These results indicate that the overexpressed GJA8 protein alters the level of LC3 and P62, which suggests that GJA8 may be involved in the process of autophagy.

The current study provides new evidence to support the GJA8 gene as a susceptibility gene for ARC in the Chinese population. In addition to interfering with the functions of gap junctions and hemichannels, GJA8 variants may also induce lens opacification by disturbing the autophagic process. Given the limited sample size and single population, further studies in other, larger populations are warranted. The relationship between GJA8 and autophagy also needs to be addressed by elucidating the interactive molecules in detail in future research.

Acknowledgements We would like to thank Dr. James Fielding Hejtmanci from Ophthalmic Genetics and Visual Function Branch, National Eye Institute, National Institutes of Health for help with the statistical analysis. And this work was supported by the National Natural Science Foundation of China (Grant no. 81670834); the National Natural Science Foundation of China (Grant no. 81371000); the Fundamental Research Funds for the Central Universities (no. 2017FZA7002); the Fundamental Research Funds for the Central Universities (no. 2018FZA7007); ZhejiangTraditional Chinese Medicine Project (no. 2013ZA080); and the Natural Science Foundation of Zhejiang Province (no. LY17H090004).

Author contributions $\mathrm{XY}$ and XP designed and performed the experiments and played a vital role in interpreting the results and writing the manuscript. XZ and YC performed the data analyses. HY, XT and YT revised the manuscript. XS contributed to the conception of the study, participated in the design of the experiments, and provided reagents and materials.

\section{Compliance with ethical standards}

Conflict of interest On behalf of all authors, the corresponding author states that there is no conflict of interest.

Open Access This article is distributed under the terms of the Creative Commons Attribution 4.0 International License (http://creativeco mmons.org/licenses/by/4.0/), which permits unrestricted use, distribution, and reproduction in any medium, provided you give appropriate credit to the original author(s) and the source, provide a link to the Creative Commons license, and indicate if changes were made.

\section{References}

Bejarano E, Yuste A, Patel B, Stout RF Jr, Spray DC, Cuervo AM (2014) Connexins modulate autophagosome biogenesis. Nat Cell Biol 16:401-414. https://doi.org/10.1038/ncb2934

Beyer EC, Ebihara L, Berthoud VM (2013) Connexin mutants and cataracts. Front Pharmacol 4:43. https://doi.org/10.3389/fphar .2013 .00043

Bourne RR, Stevens GA, White RA, Smith JL, Flaxman SR, Price H, Jonas JB, Keefe J, Leasher J, Naidoo K, Pesudovs K, Resnikoff S, Taylor HR, Vision Loss Expert (2013) Causes of vision loss 
worldwide, 1990-2010: a systematic analysis. Lancet Glob Health 1:e339-e349. https://doi.org/10.1016/S2214-109X(13)70113-X

Chylack LT Jr, Leske MC, McCarthy D, Khu P, Kashiwagi T, Sperduto R (1989) Lens opacities classification system II (LOCS II). Arch Ophthalmol 107:991-997

Congdon NG, Friedman DS, Lietman T (2003) Important causes of visual impairment in the world today. JAMA 290:2057-2060. https://doi.org/10.1001/jama.290.15.2057

Dang FT, Yang FY, Yang YQ, Ge XL, Chen D, Zhang L, Yu XP, Gu F, Zhu YH (2016) A novel mutation of p.F32I in GJA8 in human dominant congenital cataracts. Int J Ophthalmol 9:1561-1567. https://doi.org/10.18240/ijo.2016.11.05

Goodenough DA (1992) The crystalline lens. A system networked by gap junctional intercellular communication. Semin Cell Biol 3:49-58

Graw J, Schmidt W, Minogue PJ, Rodriguez J, Tong JJ, Klopp N, Illig T, Ebihara L, Berthoud VM, Beyer EC (2009) The GJA8 allele encoding CX50I247M is a rare polymorphism, not a cataractcausing mutation. Mol Vis 15:1881-1885

Hammond CJ, Snieder H, Spector TD, Gilbert CE (2000) Genetic and environmental factors in age-related nuclear cataracts in monozygotic and dizygotic twins. N Engl J Med 342:1786-1790. https:// doi.org/10.1056/NEJM200006153422404

Hammond CJ, Duncan DD, Snieder H, de Lange M, West SK, Spector TD, Gilbert CE (2001) The heritability of age-related cortical cataract: the twin eye study. Investig Ophthalmol Vis Sci 42:601-605

Ibaraki N, Chen SC, Lin LR, Okamoto H, Pipas JM, Reddy VN (1998) Human lens epithelial cell line. Exp Eye Res 67(5):577-585

Jiang JX (2010) Gap junctions or hemichannel-dependent and independent roles of connexins in cataractogenesis and lens development. Curr Mol Med 10:851-863

Jiang P, Mizushima N (2015) LC3- and p62-based biochemical methods for the analysis of autophagy progression in. Mamm Cells Methods 75:13-18. https://doi.org/10.1016/j.ymeth.2014.11.021

Klein BE, Klein R, Linton KL (1992) Prevalence of age-related lens opacities in a population: the Beaver Dam Eye Study. Ophthalmology 99:546-552

Li JY, Wang QW, Fu QY, Zhu YA, Zhai Y, Yu YH, Zhang K, Yao K (2013) A novel connexin 50 gene (gap junction protein, alpha 8) mutation associated with congenital nuclear and zonular pulverulent. Cataract Mol Vis 19:767-774

Lichtenstein A, Minogue PJ, Beyer EC, Berthoud VM (2011) Autophagy: a pathway that contributes to connexin degradation. J Cell Sci 124:910-920. https://doi.org/10.1242/jcs.073072

Liu YY, Ke M, Yan M, Guo SR, Mothobi ME, Chen QA, Zheng F (2011) Association between gap junction protein-alpha 8 polymorphisms and age-related cataract. Mol Biol Rep 38:13011307. https://doi.org/10.1007/s11033-010-0230-z

Mackay DS, Bennett TM, Culican SM, Shiels A (2014) Exome sequencing identifies novel and recurrent mutations in GJA8 and CRYGD associated with inherited cataract. Hum Genom 8:19. https://doi.org/10.1186/s40246-014-0019-6

Morishita H, Mizushima N (2016) Autophagy in the lens. Exp Eye Res 144:22-28. https://doi.org/10.1016/j.exer.2015.08.019

Pankiv S, Clausen TH, Lamark T, Brech A, Bruum JA, Outzen H, Overvatn A, Bjorkoy G, Johansen T (2007) p62/SQSTM1 binds directly to Atg8/LC3 to facilitate degradation of ubiquitinated protein aggregates by autophagy. J Biol Chem 282:24131-24145. https://doi.org/10.1074/jbc.M702824200

Patel R, Zenith RK, Chandra A, Ali A (2017) Novel mutations in the crystallin gene in age-related cataract patients from a north Indian population. Mol Syndromol 8:179-186. https://doi. org/10.1159/000471992

Ren M, Yang XG, Dang XJ, Xiao JA (2017) Exome sequencing identifies a novel mutation in GJA8 associated with inherited cataract in a Chinese family Graefes. Arch Clin Exp Ophthalmol 255:141151. https://doi.org/10.1007/s00417-016-3513-9

Sagona AP, Nezis IP, Stenmark H (2014) Association of CHMP4B and autophagy with micronuclei: implications for cataract formation. BioMed Res Int 2014:974393. https://doi.org/10.1155/2014/97439 3

Shiels HJF (2017) Mutations and mechanisms in congenital and age-related cataracts. Exp Eye Res 156:95-102. https://doi. org/10.1016/j.exer.2016.06.011

Shiels A, Hejtmancik JF (2013) Genetics of human cataract. Clin Genet 84:120-127. https://doi.org/10.1111/cge.12182

Su S, Yao Y, Zhu RR, Liang CK, Jiang SQ, Hu N, Zhou J, Yang M, Xing Q, Guan HJ (2013) The associations between single nucleotide polymorphisms of DNA repair genes, DNA damage, and agerelated cataract: Jiangsu Eye Study. Investig Ophthalmol Vis Sci 54:1201-1207. https://doi.org/10.1167/iovs.12-10940

Zhao Z, Fan Q, Zhou P, Ye H, Cai L, Lu Y (2017) Association of alpha A-crystallin polymorphisms with susceptibility to nuclear agerelated cataract in a Han Chinese population. BMC Ophthalmol 17:133. https://doi.org/10.1186/s12886-017-0529-9

Zhou Z, Wang B, Hu S, Zhang C, Ma X, Qi Y (2011) Genetic variations in GJA3, GJA8, LIM2, and age-related cataract in the Chinese population: a mutation screening study. Mol Vis 17:621-626

Zhu Y, Yu H, Wang W, Gong X, Yao K (2014) A novel GJA8 mutation (p.V44A) causing autosomal dominant congenital cataract. PLoS One 9:e115406. https://doi.org/10.1371/journal.pone.0115406 